\title{
Speckle Reconstruction with Corruption through Multimode Fibers Using Deep Learning
}

\author{
Pengfei Fan, Michael Ruddlesden, Yufei Wang, Lei Su* \\ School of Engineering and Materials Science, Queen Mary University of London, Mile End Road, London, E1 4NS \\ *l.su@qmul.ac.uk
}

\begin{abstract}
We present for the first time a deep learning approach toward speckle reconstruction with corruption through a multimode fiber (MMF) with a long length. Our experiments demonstrate that a small partly or randomly corrupted speckle can be reconstructed into its intact speckle over a $1 \mathrm{~km}$ $100 \mu \mathrm{m}$-core step-index MMF.

OCIS codes: (060.2270) Fiber characterization; (060.2330) Fiber optics communications; (200.4260) Neural networks
\end{abstract}

\section{Introduction}

Multimode fibers (MMFs) have attracted great interest for fiber optic communication due to their unparalleled information transport capabilities. However, when light is coupled into an MMF, a so-called speckle pattern is observed at the fiber output. In addition, noise exists in every communication system. Due to factors such as attenuation and dispersion of optical fibers, the modulated signal transmitted to the receiver-end becomes a weak and corrupted speckle [1]. Although it has been demonstrated that input MNIST digits and natural scenes can be retrieved from MMF output speckles through deep learning [2-4], the results reported so far are based on experiments using a stable MMF with a relatively short length. None of these assumptions satisfies the real-word settings in optical fiber communications over large distances, where signals are corrupted by noise. In this paper, we present a deep learning approach to reconstruct the corrupted speckle captured at the receiver-end after the transmission over a $1 \mathrm{~km} 100 \mu \mathrm{m}$ core step-index MMF. It is worth mentioning that the experiments reported here focus on the data processing at the receiver-end, with the purpose of exploring the transverse spatial properties of MMFs.

\section{Methods}

The experimental setup used to demonstrate the deep-learning-based corrupted speckle reconstruction framework is shown in Fig. 1. The laser beam $(532 \mathrm{~nm}, 50 \mathrm{~mW}$, Cobolt Samba) is expanded, collimated, and projected onto a digital micromirror device (DMD, ViALUX V-7001, $>20 \mathrm{kHz}$ ). The wavefront of the incident laser beam is modulated by the pattern on the DMD and is consequently coupled into the input of the $1 \mathrm{~km} \emptyset 100 \mu \mathrm{m}$ MMF (SI 100/140-22/250) using a tube lens and microscope objective. At the distal end of the fiber, a further microscope objective and tube lens are used prior to imaging the speckle pattern on a cMOS camera (QImaging optiMOS). The patterns displayed on the DMD were randomly generated to contain 100 pixels in a $10 \times 10$ configuration, with $50 \%$ each 'ON' and 'OFF' pixels. In each case, the inputs were scaled up by a factor of five, meaning one input macro-pixel comprised 25 individual micromirrors. The system operates at $200 \mathrm{fps}$. To train the deep convolutional neural network (CNN), we obtain one dataset containing 10,000 speckle images by projecting different random patterns onto the DMD.

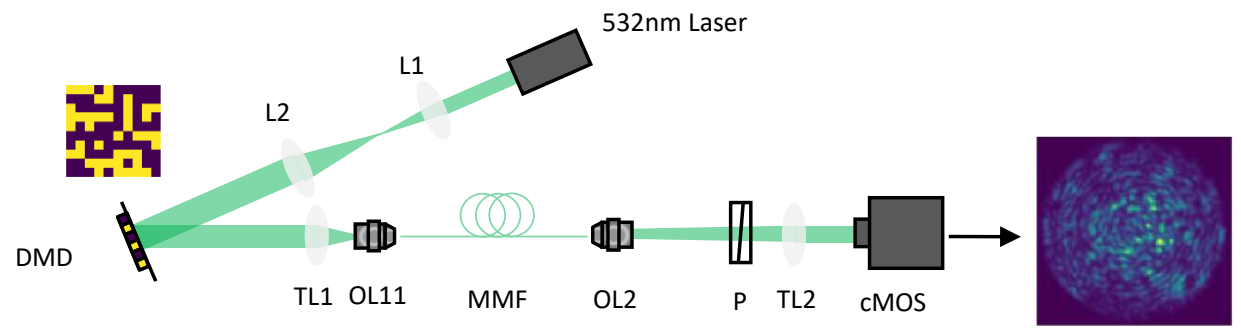

Fig. 1. Experimental setup for speckle reconstruction with corruption over a single MMF.

The intact speckles are corrupted using two types of masks comprising concentric rings and random pixels respectively. 90\% (9000) of these corrupted speckle images and their corresponding intact speckles are randomly selected for training with the remaining 1000 data pairs used to test the final CNN. As described in Fig. 2, the proposed CNN architecture follows the 'U-net' encoder-decoder structure further enhancing each convolutional layer with a dense block. To quantify the error between the predicted speckles and the original speckles, mean square error (MSE) is used. The learning rate parameter is initially set as 0.01 , and the total batch is split into mini-batches of 32 patches each. The validation loss is used to monitor early stopping and dictate the degree to which the learning rate decreases 
during training. After sufficient training, the network is blindly given a corrupted speckle pattern and rapidly outputs a predicted speckle pattern. The deep neural network is deployed under the framework of TensorFlow back-end (Google). The training phase of the network was performed using a Tesla V100 GPU card (Nvidia) and a 16 Core Xeon Gold 6142 processor (Intel) with 7.5GB RAM requested for each core.

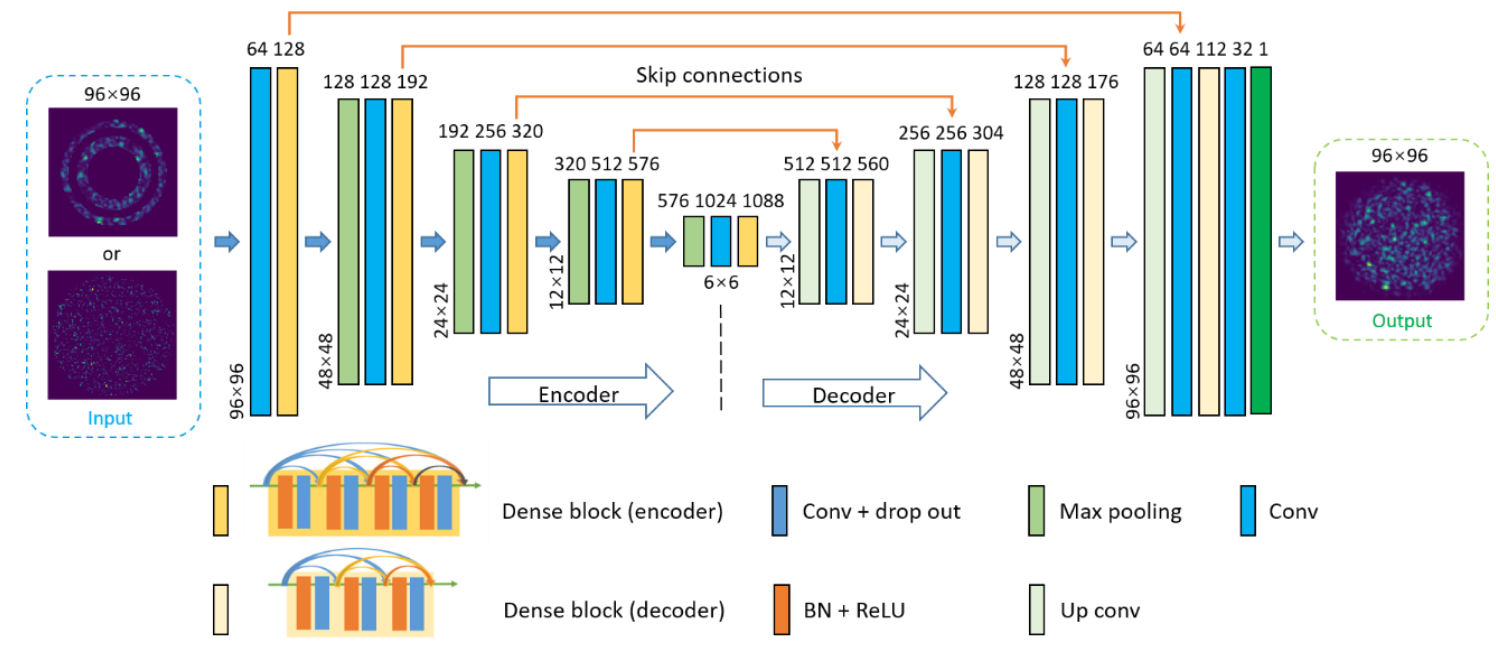

Fig. 2. The proposed CNN architecture.

\section{Results and Discussion}

When the stability of the optical system is measured for the $1 \mathrm{~km}$ MMF, the correlation between output speckles with the same input drops quickly to $20 \%$ (Fig. 3(a)). MMFs are notoriously unstable, susceptible to environmental factors such as movement caused by airflow and temperature. Despite the high instability with the $1 \mathrm{~km}$ fiber, the reconstruction with high-resolution indicates the CNN includes this instability in its learning. As plotted in Fig. 3(b), we show the process for the generation of corrupted speckles for training using masks comprising concentric rings and random pixels. Examples of predicted speckle patterns and their corresponding Pearson correlation coefficient (PCC) for different corrupting methods are also illustrated in Fig. 3(b).

(a)

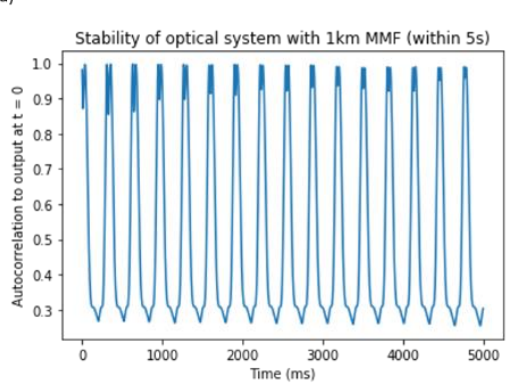

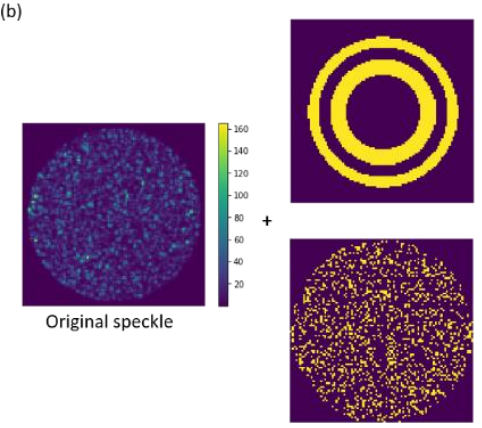

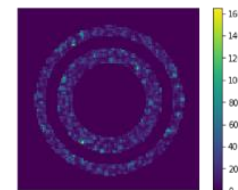

${ }_{-\infty}^{\infty 00} \stackrel{\substack{150 \\-100 \\-120 \\-100}}{\mathrm{CNN}}$

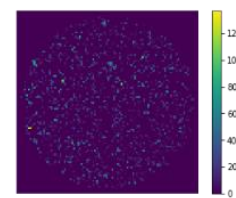

Corrupted speckle

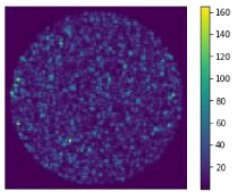

PCC: $97.96 \%$

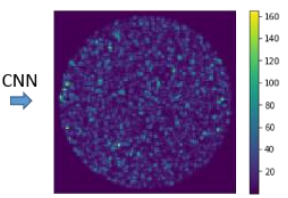

PCC: $98.31 \%$

Fig. 3. (a) Stability of the optical system including the $1 \mathrm{~km}$ MMF. A periodic fluctuation is observed. (b) Examples of predicted speckle patterns.

To conclude, we proposed a deep-learning-based corrupted speckle reconstruction framework with high-resolution over a $1 \mathrm{~km} 100 \mu \mathrm{m}$-core step-index MMF by implementing a densely connected CNN. Our experiments demonstrate that a small partly or randomly corrupted speckle can be reconstructed into its intact speckle and that almost every intact speckle includes some redundant information from the transmitted original signal. This provides a basis for the further exploration of space-division multiplexing of MMFs and the fabrication of the optical receiver in the optical fiber communication system.

\section{References}

[1] S. Bottacchi, "Noise Principles in Optical Fiber Communication," (2009).

[2] N. Borhani, E. Kakkava, C. Moser, and D. Psaltis, "Learning to see through multimode fibers," Optica 5, 960-966 (2018)

[3] P. Caramazza, O. Moran, R. Murray-Smith, and D. Faccio, "Transmission of natural scene images through a multimode fibre," Nature Communications 10, 2029 (2019).

[4] P. Fan, T. Zhao, and L. Su, "Deep learning the high variability and randomness inside multimode fibers," Optics Express 27, 20241-20258 (2019). 